Revue européenne des sciences sociales

European Journal of Social Sciences

XLII-129 | 2004

La sociologie durkheimienne : tradition et actualité

\title{
Un classique peu conformiste : la cote des prénoms
}

\section{François Héran}

\section{OpenEdition}

\section{Journals}

Édition électronique

URL : http://journals.openedition.org/ress/396

DOI : $10.4000 /$ ress.396

ISSN : 1663-4446

\section{Éditeur}

Librairie Droz

\section{Édition imprimée}

Date de publication : 1 mars 2004

Pagination : 159-178

ISBN : 2-600-00941-8

ISSN : 0048-8046

Référence électronique

François Héran, «Un classique peu conformiste : la cote des prénoms 》, Revue européenne des sciences sociales [En ligne], XLII-129 | 2004, mis en ligne le 05 novembre 2009, consulté le 19 avril 2019. URL: http://journals.openedition.org/ress/396; DOI : 10.4000/ress.396 


\title{
François HÉRAN
}

\section{UN CLASSIQUE PEU CONFORMISTE : LA COTE DES PRENOMS}

\author{
จ "Allez, Ketin, \\ Gonathan Jesus, \\ Jessica Deborah, \\ Maria Sheila, \\ habillez-rous! on va \\ d la manifestation \\ antimondialisation ? $^{\text {st }}$ \\ Ster la pancarte : \\ "Non à la \\ glabatisation". Dessin \\ d'Idigoras y Pachi \\ paru dans \\ El Mundo, Madrid.
}

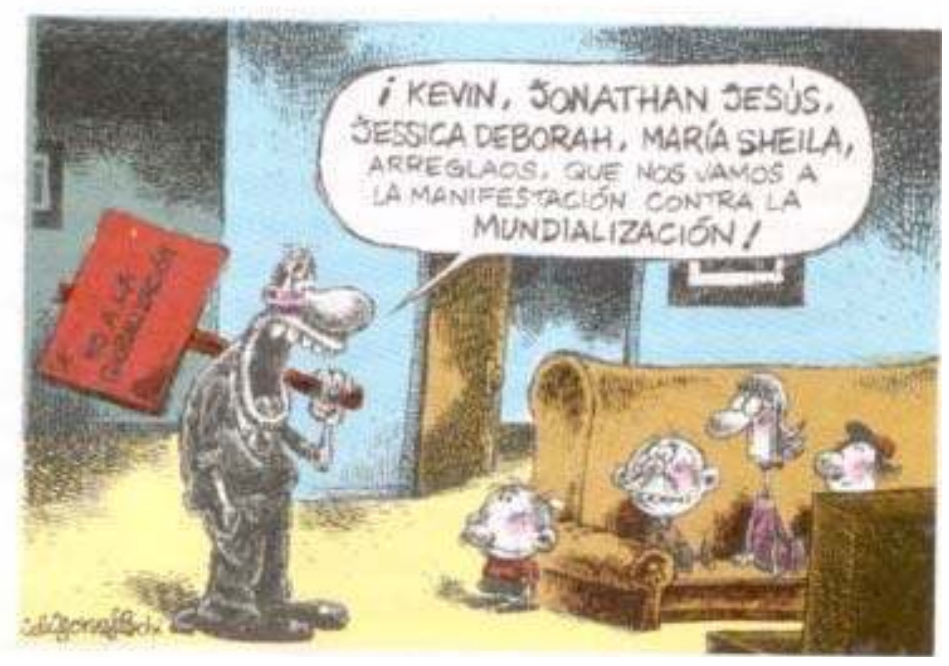

Dessin d'Idigoras y Pachi, El Mundo, Madrid, reproduit dans Courrier international, 31 janvier 2002. 
Les initiateurs du présent recueil m'invitent à revenir en toute liberté sur ce grand classique qu'est devenu La cote des prénoms. Je leur en suis reconnaissant car je tiens cet ouvrage de Philippe Besnard et Guy Desplanques pour un chefd'œuvre. J'avais salué sa parution en 1986 par un compte rendu circonstancié dans la Revue française de sociologie. J'avais aussi partagé leur indignation quand ils découvrirent un beau jour de 1994 qu'une grande revue de sociologie américaine avait publié un article qui pillait ce travail sans vergogne. L'auteur avait opportunément oublié qu'il avait rencontré Philippe Besnard à Paris et obtenu de lui de précieux conseils de méthode. Le détournement était si flagrant que la revue accepta de publier un rectificatif (Besnard 1995). Cet incident n'a pas empêché le traité de Besnard et Desplanques de poursuivre sa carrière et de devenir une référence internationale reconnue.

Ma conviction n'ayant fait que se renforcer avec le temps, je voudrais revenir ici sur les raisons de mon admiration. Mes réflexions n'apporteront rien de neuf aux plus fidèles des lecteurs, et encore moins aux auteurs. Elles ont pour seul objectif d'inviter mes jeunes collègues au plaisir de la découverte.

On ne dira jamais assez combien «le» Besnard-Desplanques est un objet singulier dans la production sociologique. Dès sa première édition, sous le titre $U n$ prénom pour toujours: la cote des prénoms hier, aujourd' hui et demain, il faisait coup triple: il comblait une lacune criante dans l'analyse scientifique d'une pratique pourtant universelle et familière, il touchait un large public par une vulgarisation de haut niveau et il offrait aux enseignants de sociologie un matériel idéal pour initier les étudiants à l'approche quantitative des phénomènes sociaux. Trois niveaux de lecture - et, en définitive, la statistique sociale au service de tous. Je ne connais pas d'autre exemple d'une telle réussite dans la discipline.

La clef du succès tient d'abord à la complémentarité des deux auteurs: un sociologue cultivé et féru de chiffres qui fait alliance durable avec un statisticiendémographe tout ouvert à la science sociale, ce n'est pas si fréquent. Ensemble, les deux chercheurs tenaient les deux bouts de la chaîne: d'un côté, le programme théorique annoncé par Besnard dès 1979 dans un article visionnaire; de l'autre, la construction d'une colossale base de données produite dans le cadre de la statistique publique: elle dépassait déjà deux millions de naissances en 1986, elle en rassemble désormais près de seize ${ }^{1}$.

\section{UNE APPROCHE DOUBLEMENT LONGITUDINALE}

Une autre qualité de l'ouvrage est apparue au fil des éditions successives: son installation dans la durée. Voilà plus de quinze ans que chaque année, à l'automne,

L'Insee vend sa base de données aux chercheurs au prix coûtant. Pour éviter les conflits d'intérêts entre production publique et usage privé, les auteurs de La cote des prénoms l'acquièrent chaque année aux mêmes conditions que tout autre acheteur, bien que l'un d'eux exerce sur place son métier de démographe. Signalons que les revues régionales de l'Insee, s'inspirant du modèle fourni par l'ouvrage, ont publié plusieurs analyses régionales, parfois couplées avec l'étude des patronymes (par exemple Moreau et al. 1996). La presse quotidienne régionale s'en est souvent fait l'écho, ce qui a sans doute constitué un relais efficace à la diffusion des travaux de Besnard et Desplanques. 
paraît une version mise à jour: la maquette est renouvelée, les données chiffrées sont actualisées, le commentaire s'enrichit de développements inédits (le dernier en date, celui de l'édition millésimée 2004 publiée en septembre 2003, contient une analyse de la migration régionale des prénoms, illustrée par une éloquente série de cartes) et l'on sent combien l'expertise accumulée permet aujourd'hui aux auteurs de déjouer sans peine toutes sortes de pièges dans l'analyse des données (ainsi, le maintien d'un prénom à un score élevé au niveau national ne signifie pas forcément qu'il s'agit d'un classique stable; il peut cacher une rapide circulation à l'échelle régionale, qui en change la signification). Pour qui a tenté de suivre ce travail d'actualisation d'une édition à l'autre, le BesnardDesplanques est devenu quelque chose d'unique: un véritable livre permanent, le fruit toujours renouvelé d'une recherche qui vit et respire en même temps que son objet.

On dira que c'est chose commune chez les économistes ou les démographes, accoutumés à suivre la conjoncture dans des publications périodiques. On rappellera aussi que les chroniqueurs gastronomiques ne font pas autre chose quand ils publient chaque année des palmarès actualisés. Et il est vrai que La cote des prénoms s'inscrit aussi dans le genre de la chronique conjoncturelle ou dans celui du guide ou du palmarès pour consommateurs éclairés. Mais elle va évidemment plus loin. Loin de la routine des analyses de conjoncture, l'approche adoptée est doublement longitudinale. À la fois rétrospective, puisqu'elle retrace l'évolution du choix des prénoms depuis au moins le début du $\mathrm{XX}^{\mathrm{e}}$ siècle, et prospective, puisque les auteurs suivent en temps réel la conjoncture de chaque prénom et n'hésitent pas à la projeter pour les années à venir, non sans prendre quelques risques, offrant ainsi au lecteur attentif la possibilité de vérifier le degré de réalisation des prédictions dès l'édition suivante (disons-le d'emblée: le taux de réalisation est remarquablement élevé !).

Bien entendu, la dimension longitudinale se double d'une dimension transversale: à la mesure des flux annuels, Besnard et Desplanques ajoutent la mesure actualisée des stocks. Et cette fois, c'est le langage des épidémiologistes qui vient à l'esprit: La cote des prénoms livre aussi bien l'incidence d'un prénom (sa probabilité d'apparition dans l'année) que sa prévalence dans l'ensemble de la population (c'est-à-dire la place occupée par chaque prénom dans le stock accumulé au sein de la population en général, toutes générations confondues). «Transversal», «longitudinal», «rétrospectif», «prospectif», « incidence», «prévalence»: inutile de dire que ce jargon est rigoureusement banni par nos rédacteurs, qui tiennent à rester accessibles, mais ce refus autorise justement des usages pédagogiques intéressants car un public d'étudiants peut utilement se pencher sur ces pages pour essayer d'identifier les différentes techniques d'approche mises en œuvre.

\section{DE MÉMOIRE D'HOMME : DES CYCLES D'UN SIÈCLE ET DEMI}

D'une édition à l'autre, le commentaire rédigé par Philippe Besnard tente de progresser dans l'identification des cycles de la mode qui gouvernent le choix des prénoms. Résultat surprenant: il apparaît de plus en plus qu'il s'agit d'une mode à récurrence extrêmement longue. Un siècle et demi sépare les sommets des deux 
courbes de popularité successives d'un même prénom. Ainsi, nous dit Besnard, Sophie culmine dans les années 1820 et les années 1970, Julie dans les années 1830 et 1980, Pauline vers 1850 et 1995, tandis que Victor, prénom à succès vers 1860, vient seulement de revenir au sommet. À ce rythme, il est vain d'espérer le retour de Marcel avant quelques décennies, puisqu'il était à la mode entre 1900 et 1924 (La cote...2004, pp. 316 et 219).

La première de ces pointes nous est connue par le travail de Jacques Dupâquier, Le temps des Jules (1987), qui avait analysé l'évolution de 90000 prénoms au cours du XIX ${ }^{\mathrm{e}}$ siècle, avant de confier généreusement son fichier à Philippe Besnard. Pour scruter la conjoncture annuelle des choix de consommation, il faut, en effet, regarder loin, très loin en arrière. Par ce double regard, porté au plus près comme au plus loin, Besnard et Desplanques confirment à quel point la sociologie bien comprise est une histoire du temps présent. Mais une moyenne de cent cinquante ans entre les succès successifs du même prénom ne laisse pas d'être troublante: est-ce encore une durée à portée d'homme? L'intervalle excède de loin l'espérance de vie maximale de chacun, pour ne pas parler de l'espérance de vie du chercheur attelé au suivi longitudinal de son fichier: condamné à vieillir en même temps que ses données, il doit attendre que le temps passe pour mesurer l'œuvre du temps.

De ce que le retour des prénoms survienne au-delà d'une vie d'homme, doiton conclure à l'existence de forces collectives qui actionneraient les intéressés à leur insu? Besnard ne nous oriente pas dans cette direction. L'adoption des prénoms se fait de proche en proche, d'un groupe social au groupe immédiatement voisin. Les cartes régionales introduites dans l'édition 2004 (p. 364) montrent la propagation d'un prénom comme Loïc, qui émerge en Bretagne au début des années cinquante et met quarante ans à gagner les frontières est et sud du pays. C'est le signe que les emprunts, quand ils ne sont pas centralisés par la télévision, suivent les chemins ordinaires de la sociabilité et de l'émulation sociale. En définitive, les analyses de Besnard suggèrent qu'un laps de cent cinquante ans pourrait bien correspondre à la somme de deux durées, dont chacune reste à échelle humaine. Entre l'émergence et la disparition d'un prénom à la mode (mesurées par le franchissement du seuil de $2 \%$ des naissances annuelles de même sexe) s'écoule d'abord en moyenne une quarantaine d'années, pendant lesquelles chacun peut percevoir tour à tour l'émergence, la diffusion et la vulgarisation d'un prénom. Puis intervient, selon une métaphore chère à Besnard, un «purgatoire » incompressible d'un peu plus d'un siècle, qui correspond au temps nécessaire à l'extinction définitive de toute une génération.

Ainsi, loin d'expliquer la remontée cyclique des prénoms par un mystérieux retour collectif du refoulé, Besnard nous invite à penser que ce serait le souvenir vivant des prénoms portés par la génération des grands-parents, voire des arrière grands-parents, qui viendrait sciemment «refouler le retour». Il met le doigt, ce faisant, sur une caractéristique majeure de nos sociétés: alors que dans la société traditionnelle, le prénom s'héritait des grands-parents par le biais du parrainage spirituel, nous attendons désormais la disparition complète des anciens pour récupérer leurs prénoms, comme s'il fallait éviter à tout prix le chevauchement ou, peut-être, la concurrence onomastique des générations. La recherche de l'originalité ne s'observerait pas seulement sur la dimension horizontale qui éparpille les familles et les groupes mais sur l'axe vertical où se succèdent les générations. 
C'est une découverte majeure. Il serait intéressant de pouvoir vérifier un jour si l'allongement très sensible de l'espérance de vie dans les dernières décennies a contribué à différer d'autant le retour des prénoms. Pour l'instant, cette vérification semble hors de portée, tant elle requiert de temps.

\section{LA DIFFUSION VERTICALE DES GOÛTS : UN MODÈLE BIEN ATTESTÉ...}

Un autre résultat majeur de La cote des prénoms est la preuve empirique qu'un prénom a beau être choisi pour sa beauté ou rejeté pour sa laideur, il n'est, selon la formule de Besnard, «ni beau ni laid en soi». Aussi étrange que cela nous paraisse aujourd'hui, des générations entières de parents ont pu voir en Marcel, Eugène, Marie-Louise ou Germaine le plus beau des prénoms. A son tour, la génération du baby-boom découvre, non sans douleur, que Michel, Martine ou Patrick sont devenus trop désuets pour qu'on songe aujourd'hui à les donner aux nouveau-nés, sous peine de leur infliger un sérieux coup de vieux. Ce ne sont pas les prénoms qui sont en cause, conclut Besnard, mais le fait de les choisir à tel ou tel moment. Traduisez: le fait de les choisir avant, avec ou après le choix des autres. Nous ne jugeons directement de rien, nous en jugeons par rapport au jugement d'autrui.

Cette proposition serait bien générale et, pour tout dire, assez banale si Besnard ne prenait soin de pousser l'analyse (La cote... 2004, p. 318). Il montre que trois conditions doivent être remplies pour qu'un prénom tombe en disgrâce. On peut les reformuler ainsi:

- un prénom nous paraît d'autant plus laid qu'il a été jugé très beau dans le passé (le critère étant l'attribution à une forte proportion de naissances, de l'ordre d'au moins une sur cinquante);

- un prénom est d'autant plus répulsif que sa dernière période attractive a été brève;

- un prénom semble irrémédiablement déclassé quand ses porteurs les plus visibles ont de 40 à 90 ans, c'est-à-dire sont entrés dans la seconde moitié de leur vie.

Ainsi, l'amour que l'on a pour un prénom finit rapidement par l'étouffer s'il est trop intense et trop bref. C'est ce qui fait tout à la fois la force et la faiblesse des engouements collectifs: leur légitimité vite acquise est vulnérable, parce que la poussée des nouveaux venus n'est pas moins légitime et suffit à les dépouiller de leur nouveauté.

En rapprochant les diverses analyses publiées par Besnard et Desplanques séparément ou conjointement, on peut dégager trois genres fondamentaux de prénoms : les «prénoms-mode», qui se diffusent de haut en bas, les prénoms «classiques » cultivés de façon stable par les familles bourgeoises et les prénoms d'origine américaine que les milieux populaires empruntent directement aux médias.

Les «prénoms-mode», dans la terminologie de Besnard, sont les prénoms qui sortent un jour de l'ombre pour franchir un seuil de visibilité situé aux environs de 
$2 \%$ des naissances de l'année. Ils se propagent ensuite de proche en proche (ce qui donne mécaniquement à leur progression la forme d'une courbe log-normale), avant de décliner et disparaître. Dans son article pionnier de 1986, Desplanques a retracé rétrospectivement sous forme de courbes l'ascension et le déclin de Jean, Michel, Philippe, Patrick, Christophe, Sébastien, Nicolas, qui se sont succédé au palmarès des prénoms des années trente aux années quatre-vingt. Il a fait de même pour Jeannine, Monique, Danièle, Françoise, Martine, Sylvie, Nathalie, Sandrine. Une fois reconstitués ces parcours, nos deux auteurs ont pu suivre, d'une édition à l'autre, la noria des «prénoms-mode» qui ont pris le relais: Aurélie (19811986), Élodie (1988-1990), Marine (1991-1992), Laura (1993-1994), Manon (1995-1996) et Léa (depuis 1997), la série masculine alignant quant à elle Julien (1983-1988), Kevin (1989-1994), à nouveau Nicolas (1995), puis Thomas (19962000) et Lucas (depuis 2001).

Un acquis majeur des travaux de Besnard et Desplanques est d'avoir apporté la preuve empirique que les fluctuations de la mode obéissent d'abord au principe de la diffusion verticale des goûts. On peut même dire qu'il n'en existe pas de démonstration plus solide dans la littérature sociologique. Guy Desplanques l'a montré dès son article de 1986: quand on trace les courbes de diffusion des «prénoms-mode» par catégorie sociale, elles s'alignent comme à la parade. Les cadres ouvrent la marche, suivies des professions intermédiaires, des employés et des ouvriers. Les artisans et commerçants figurent en position médiane, tandis que les agriculteurs ferment le ban. Ce sont autant de courbes normales régulièrement décalées dans le temps. Or la diffusion verticale des goût est à l'espace social ce que la propagation de proche en proche est à l'espace géographique. Et les deux phénomènes peuvent s'additionner sans contradiction.

\section{... CONFIRMÉ PAR L'ÉCHELLE SOCIOPROFESSIONNELLE DES INNOVATIONS...}

Besnard et Desplanques poussent la démonstration dans une analyse publiée en 1999 par la Revue française de sociologie. Ils utilisent cette fois les catégories socioprofessionnelles détaillées à deux chiffres. Ils prennent pour indicateur d'innovation la capacité à choisir un prénom au moins dix ans avant qu'il ne franchisse le seuil de 1 pour mille. Il en résulte une hiérarchisation des catégories sociales d'une remarquable cohérence. Les plus précurseurs sont les journalistes et les artistes, capables de lancer très tôt des prénoms inédits comme Nathalie ou Céline, exhumés dès la fin des années quarante et le début des années cinquante, alors que la diffusion de ces prénoms ne culminera en France qu'une vingtaine d'années plus tard. Ils sont immédiatement suivis des professions libérales, qui se spécialisent davantage dans la relance précoce de prénoms traditionnels comme Isabelle ou Catherine. Viennent ensuite - et ensuite seulement - les chefs d'entreprise, les cadres et les ingénieurs, ce qui indique clairement que la recherche d'un prénom précurseur est une activité de type culturel: c'est davantage l'affaire des groupes sociaux les plus instruits que celle des groupes les plus riches. Quand on passe aux professions intermédiaires, le taux d'innovation reste encore élevé chez les infirmières et les travailleurs sociaux. Au sein des indépendants, la diffusion précoce des prénoms est plus fréquente chez les commerçants et les employés de 
commerce que chez les artisans. Après quoi l'on arrive aux gros bataillons des employés, puis des ouvriers, où les catégories les moins bien placées sont les ouvriers les moins qualifiés, qu'ils travaillent dans l'industrie, l'artisanat ou l'agriculture.

Ainsi, le modèle de diffusion de haut en bas n'a rien d'un mythe, tout au contraire. Et l'article de Besnard et Desplanques vient démentir, par une démonstration impeccable, les propos hâtifs des sociologues ou des essayistes qui croient régulièrement pouvoir enterrer les catégories socioprofessionnelles. Dans un domaine comme celui de la circulation des prénoms-modes, leur pouvoir descriptif reste intact.

Reste à passer de la description à l'explication. La catégorie sociale est un faisceau de propriétés sociales agissantes qu'il n'est pas facile de démêler. On manque pour l'instant d'analyses statistiques multivariées qui, telle la régression logistique, permettraient d'en savoir plus, et c'est là assurément la principale lacune de cet ensemble de travaux. Besnard avance une hypothèse plausible qui reste à vérifier: l'innovation est d'autant plus intense que la catégorie sociale est riche en sociabilité. On sait effectivement (en particulier par l'enquête Contacts menée à l'Insee dans les années quatre-vingt) que les classes populaires ont un réseau de sociabilité fréquenté de façon intense mais répétitive et, de ce fait, plutôt pauvre en informations nouvelles. À l'inverse, la sociabilité des intellectuels et des professions libérales est faite de contacts rapides et diversifiés, propices à la diffusion des nouveautés culturelles. Cela expliquerait que les professions les plus «relationnelles » soient aussi les plus aptes à innover en matière de prénoms.

\section{... MAIS CONCURRENCÉ PAR LE MODÈLE DE LA POLARISATION DES GOÛTS}

Ces conclusions doivent cependant rester provisoires car la découverte capitale est que la diffusion en cascade n'est pas l'unique modèle de référence. À côté des prénoms soumis au cycle de la mode, La cote des prénoms signale l'existence d'un stock de prénoms «classiques»: prénoms stables, inscrits de longue date dans le patrimoine onomastique des familles bourgeoises, comme peuvent l'être Agnès, Anne, Claire, Emmanuelle, Hélène pour les filles, François, Pierre, Marc, Antoine, Bertrand, Étienne, Xavier pour les garçons.... Quasiment indémodables, relativement protégés des fluctuations sociales, ces prénoms tapissent en quelque sorte notre système d'appellation et sont disponibles pour d'éventuelles réactivations. Sur ce point précis, les analyses des auteurs ont quelque peu évolué. À lire les dernières éditions du guide, on comprend mieux que la frontière entre prénoms classiques et prénoms-mode n'est pas étanche. Il arrive aux prénoms classiques d'être emportés un temps par la mode, mais sur des vagues moins brutales et plus longues, suivies d'une retombée qui n'est jamais synonyme de disparition et ne compromet guère leur image. Ainsi Claire a-t-il progressé au tournant des années quatre-vingt, pour culminer modérément pendant une douzaine d'années au niveau de $0,8 \%$ des naissances féminines, ce qui préserve ses chances de remonter dans un avenir proche.

Très clairement, les prénoms classiques sont conformes au «goût bourgeois ». Les professions libérales y ont recours quatre à six fois plus souvent que les 
ouvriers, la distance ayant même tendance à se creuser quand le prénom se fait plus rare (La cote... 2004, p. 334). Écarts considérables, par conséquent. L'analyse à laquelle s'est livré Besnard à partir des travaux de Cyril Grange sur le Bottin mondain permet d'approfondir cette relation. Elle présente le grand intérêt de couvrir près de deux siècles de naissances, de 1820 à 1991. Il apparaît qu'avant la Grande Guerre, la population mondaine pouvait devancer la population ordinaire d'une trentaine d'années, voire davantage, dans l'innovation en matière de prénoms (l'indicateur retenu étant l'intervalle qui sépare les sommets respectifs des deux courbes). C'était le cas pour des prénoms comme Marguerite, Yvonne, Suzanne ou Madeleine, ou, côté masculin, pour Georges, Henri, Marcel, Maurice.. Depuis lors, l'avance n'a cessé de fondre: une douzaine d'années dans les années trente, moins d'une dizaine dans les années soixante, l'écart étant quasiment aboli dans les dernières années.

À ce stade de la démonstration, le lecteur pourrait conclure à l'uniformisation des goûts, mais c'est mal connaître notre sociologue, qui nous réserve encore une de ces surprises dont il a le secret: «car à mesure que les écarts se réduisent, les différences se creusent» (Besnard et Grange, 1993: 275). En effet, les prénoms adoptés par les fractions mondaines de la bourgeoisie n'appartiennent plus au même stock que ceux qui attirent le commun des mortels. Talonnée par les nouveaux bourgeois, la vieille et haute bourgeoisie du Bottin se réfugie plus que jamais dans l'univers ouaté des prénoms à cycle lent, hérités de la tradition, classiques entre les classiques, tels Antoine, Bertrand, Guy, Henri, Anne, Béatrice, Claire ou Geneviève. C'est dans ces milieux que la répulsion est naturellement la plus vive à l'égard des nouveautés anglo-saxonnes: Audrey, Vanessa, Mickaël, Kevin, etc., sont totalement bannis. Mais, plus en amont déjà, les vagues d'innovation incarnées dans les années trente par la série Yvette, Paulette, Georgette, ou à la fin des années soixante par les Sandrine, Nathalie, Corinne, étaient frappées du même ostracisme. L'aristocratie, quant à elle, n'a fait qu'accentuer les stratégies de repli de la haute bourgeoise. Les deux mondes ont en commun, non seulement de rester imperméables aux innovations populaires, mais, réciproquement, de ne guère exporter leurs prénoms les plus typés. Les Charles-Henry, CharlesÉdouard ou Jean-Eudes apparaissent trop marqués pour espérer séduire les autres catégories sociales.

Mais, s'il y a des «prénoms bourgeois » ou hautement bourgeois, il y a aussi des «prénoms populaires», en ce sens qu'ils ne résultent pas de la retombée en cascade des choix effectués par les catégories supérieures mais appartiennent au peuple et à lui seul. Ce sont les fameux prénoms à consonance anglo-saxonne, tirés des télé-feuilletons américains. En 1986, Besnard et Desplanques relevaient l'apparition en milieu populaire de prénoms tels que Vanessa, Cindy, Jennifer, Audrey, Anthony, Christopher, Michaël, David, Grégory... Par la suite, ils ont vu surgir Kevin, Allan, Jason, Elliott, Brenda, Kimberley, Stacy, Wendy et bien d'autres. L'analyse régionale montre que ces innovations éclosent souvent au sein de régions ouvrières comme le Nord-Pas-de-Calais ou la Picardie. Faute d'être relayées par les classes moyennes ou supérieures ou par les agriculteurs, leur diffusion reste limitée aux milieux populaires. Mais comme ces derniers pèsent beaucoup dans la population globale, il s'ensuit qu'une part considérable des choix de prénoms en France s'effectue en dehors du schéma classique de la diffusion du haut vers le bas. 
Besnard conclut à l'existence d'une «polarisation» des goûts entre «goûts bourgeois » et «goûts populaires » qui, loin de s'atténuer, tend à se creuser avec le temps. Le fossé d'ignorance qui sépare les deux pôles est tel qu'on peut imaginer, à la limite, l'essor simultané du même prénom - ou de deux prénoms homonymes - dans les deux mondes. Et tel est bien le cas, semble-t-il, du prénom féminin Inès (La cote... 2004, pp. 177 et 341), dont Besnard signale l'étonnante double vie: substitut chic d'Agnès dans les milieux BCBG (il est en tête du Bottin Mondain en 2000-2002), c'est aussi une variante du prénom arabe Inas, qui connaît actuellement une grande vogue dans les banlieues. Il cumule en 2002 le premier rang en Seine-Saint-Denis et le troisième dans les Hauts-de-Seine et à Paris, si bien qu'il arrive déjà en septième position du palmarès national féminin! Cas exceptionnel, précise Besnard, mais d'autant plus fascinant. Qu'adviendra-t-il lorsque les hautslieux du 92 finiront par découvrir (grâce à la dernière édition du guide...) cette fâcheuse homonymie avec les bas-fonds du 9-3? Vont-ils précipiter la chute d'Inès pour éviter la confusion? Pour lever ce suspense sociologique, réservez dès maintenant chez votre libraire l'édition 2005 de La cote des prénoms.

\section{BESNARD ET BOURDIEU}

Pour en revenir à des considérations académiques plus traditionnelles, les validations empiriques de la théorie des goûts et de leur diffusion amèneront certains lecteurs à se demander comment se situe exactement l'approche de Besnard par rapport à la théorie de la distinction promue par Bourdieu. Â vrai dire, on pourrait aussi bien inverser la question car l'article programmatique de Besnard, publié dès 1979, est de la même année que La distinction. Plus généralement, Besnard n'a jamais considéré comme une nécessité vitale d'avoir à se situer par rapport à Bourdieu, tandis que ce dernier s'est bien gardé, dans La distinction ou ailleurs, d'aborder un terrain qu'il savait aussi bien labouré par son collègue. On y voit plus clair, me semble-t-il, si l'on distingue au moins deux strates dans la théorie de Bourdieu.

L'une exploite un filon structuraliste, qui plonge ses racines dans la fameuse théorie du symbole développée par Durkheim dans Les formes élémentaires de la vie religieuse, selon laquelle la société est capable de valoriser à l'extrême à peu près n'importe quoi, y compris des supports sans signification intrinsèque (Durkheim cite le drapeau, le timbre-poste, les outils rituels, les emblèmes claniques). Ces objets se retrouvent chargés de toute l'autorité du corps social. D'une certaine façon, la théorie de Bourdieu revient à reporter sur un groupe dominant (idée qui s'alimente de la double lecture de Marx et de Weber) le pouvoir d'investissement dévolu par Durkheim à la société tout entière. La diffusion verticale des goûts manifesterait la capacité qu'auraient les dominants à consacrer des jugements esthétiques arbitraires s'imposant au reste du corps social.

La deuxième strate théorique est plus proche d'une idée commune, développée de longue date par les moralistes français ou anglais et reprise par des auteurs aussi remarquables que Veblen ou Goblot (que La distinction, distinction oblige, ne cite jamais). Elle pose que les innovations se diffusent de haut en bas dans le corps social, avec un double mouvement: les groupes supérieurs sont 
menacés de rattrapage par les groupes immédiatement inférieurs qui cherchent à les imiter. En compensation, ils tentent à s'en démarquer dès qu'ils voient les biens diffusés se vulgariser.

Besnard, très clairement, n'adhère pas à la théorie de la diffusion de l'arbitraire par les dominants mais il ne fait aucun doute qu'il conforte la théorie générale de la diffusion verticale des goûts, tout en lui posant des limites bien marquées, grâce au constat de la polarisation. Il démontre, données à l'appui, qu'on peut tout à la fois défendre l'idée que les jugements de goût, en ce domaine comme en d'autres, sont socialement construits, et récuser la théorie selon laquelle les dominants parviendraient à imposer l'arbitraire de leurs jugements. En réalité, il s'en faut de beaucoup que les innovations lancées par les catégories dominantes imprègnent toutes le reste du corps social.

\section{L'AMBIVALENCE DU MODÈLE AMÉRICAIN}

On objectera peut-être que l'importation massive de prénoms américains est encore le signe d'une dépendance vis-à-vis d'un pouvoir dominant, celui de l'industrie culturelle américaine. Et que, réciproquement, le temps où la France parvenait à exporter aux États-Unis des prénoms tels que Michelle, Jacqueline, Nicole ou Renée est bien révolu, si l'on en juge par les palmarès américains reproduits par Besnard: la balance des échanges onomastiques est devenue fortement déficitaire à notre détriment. On dira aussi que l'attrait pour les prénoms angloaméricains est inversement proportionnel à la pratique effective de la langue anglaise, telle qu'on peut en mesurer le dégradé social dans le volet linguistique de l'enquête Famille de 1999. Le choix de prénoms anglo-américains serait davantage le choix de l'ignorance qu'une importation sélective et maîtrisée.

Mais il n'est pas sûr que ces jugements de valeur saisissent bien le fond de la chose. Les feuilletons américains sont un réservoir commun de prénoms, comme peuvent l'être les arbres généalogiques et les souvenirs de famille dans les milieux favorisés. Le peuple innove autant que la bourgeoisie, sinon davantage. Et s'il est vrai que l'innovation des intellectuels bien nés semble tenir à la faculté d'utiliser les informations nouvelles venues de la périphérie de ses réseaux de relations (selon le schéma de la «force des liens faibles» développé par Granovetter), on peut voir un équivalent de cette capacité dans la libre exploitation que fait le peuple des spectacles exotiques fournis à domicile par la télévision.

Dans ces conditions, les emprunts aux feuilletons américains peuvent difficilement s'interpréter comme le signe d'une soumission culturelle. À ceux qui redoutent les effets d'une mondialisation incontrôlée des prénoms, les palmarès des pays étrangers recueillis par Besnard depuis quelques années apportent plutôt des motifs d'espoir. L'influence américaine s'exerce encore de façon très variable. Malgré les bons scores de Kevin, Jennifer, ou Vanessa au début des années quatrevingt-dix, le palmarès de l'Allemagne est revenu plus récemment vers le fonds germanique: Alexander, Lukas, Maximilian, Julia, Katharina... Les prénoms du Québec francophone ne forment pas le même tableau qu'en France, en partie par résistance explicite à l'américanisation. Enfin, le palmarès américain des prénoms se renouvelle lui-même à un rythme plus lent que le palmarès français et, de surcroît, aligne des prénoms très différents des nôtres, surtout pour la partie 
masculine (Jacob, Michael, Joshua, Matthew, Ethan, etc.). Chaque pays reste encore largement pris dans son propre marché.

Ce qui frappe en définitive, c'est l'impuissance patente de la bourgeoisie française à imposer ses choix onomastiques aux milieux populaires. On peut même douter qu'une telle volonté d'imposition existe. En préférant au modèle dominant national un modèle transatlantique omniprésent, le peuple exerce sa faculté de choix avec les moyens du bord mais aussi avec une belle insouciance et une tranquille obstination. Il y a longtemps que ces choix exotiques désespèrent nos intellectuels. Victor Hugo, déjà, caractérisait les Thénardier dans Les misérables par le fait qu'ils avaient prénommé leurs filles Éponine et Azelma sous l'inspiration de quelque mauvais feuilleton d'outre-Manche. Il expliquait ce dédain pour le fonds traditionnel de nos prénoms par une mentalité de «déclassés » (entendez par là les déracinés du peuple, en quête d'ascension sociale dans la ville).

Mais Hugo était plus poète que sociologue. Plutôt qu'une volonté exacerbée d'échapper à sa condition, le choix d'un prénom exotique pourrait bien traduire un désir d'affranchissement ou d'émancipation par rapport aux choix convenus des «gens comme il faut». C'est une façon pour le peuple de rester lui-même - de la même façon que les Bretons pensent rester eux-mêmes en optant pour des prénoms bretons. Se greffe là-dessus une question subsidiaire évoquée rapidement par Besnard et Desplanques: les milieux populaires comprenant désormais une forte composante immigrée, on peut se demander, par exemple, si le choix de prénoms d'origine arabe n'est pas en train d'évoluer vers des formes mixtes, où l'américanisation et l'arabisation vont de pair grâce à des prénoms comme Sami ou Samy, Sara ou Sarah, qui sont les analogues de la world-music dans le monde des prénoms. Peut-être la francisation d'Inas en Inès accompagne-t-elle la progression des mariages mixtes. Malheureusement, les données ne sont pas encore assez abondantes pour que l'on puisse aujourd'hui vérifier ce genre d'hypothèses ${ }^{2}$.

Quelle est la position de Besnard sur la question spécifique de l'américanisation? Il n'hésite pas à sortir de la neutralité qui sied au sociologue pour émettre à ce sujet quelques jugements de valeur. Son point de vue est celui de l'intérêt de l'enfant, dont il ne faut jamais oublier qu'il deviendra adulte et devra subir toute sa vie les conséquences d'un prénom mal choisi. Ce thème est depuis le début un fil conducteur du commentaire de Besnard (on se rappelle le titre initial de l'ouvrage, Un prénom pour toujours). Le prénom est un atout durable pour l'enfant, d'aucuns diraient un capital, susceptible de se dévaluer très vite, quand ce n'est pas d'entrée de jeu. Il peut donner à l'enfant, selon le cas, un coup de vieux ou un coup de jeune ${ }^{3}$. Il risque aussi de signaler à tout jamais l'appartenance à un milieu

Besnard et Desplanques relèvent la difficulté qu'il y a à suivre l'évolution des prénoms d'origine immigrée. Les naissances de mère étrangère représentent seulement 10 à $11 \%$ des naissances annuelles depuis vingt ans, et seule une fraction d'entre eux s'accompagnent du choix d'un prénom étranger. De ce fait, Mehdi et Mohammed, qui sont les prénoms étrangers les plus fréquents en France, ne concernent chacun que $0,4 \%$ des naissances.

3 J'ai eu l'occasion de fréquenter un jeune collègue prénommé Albert, qui devait à son prénom le douteux privilège de recevoir régulièrement des publicités l'invitant à souscrire un contrat-dépendance ou à préparer ses obsèques. L'exploitation des fichiers de l'Insee ou des méga-bases de clientèles avait permis aux spécialistes du marketing onomastique d'associer les Albert aux générations de l'entre deux guerres. 
peu instruit, où les origines antiques ou bibliques du prénom sont ignorées (Chloé écrit Cloé, Théo écrit Téo, voire Théau, Timothée écrit Timothé, etc.) ou qui se fourvoie dans l'écriture d'un prénom anglais par un effet d'hypercorrection (Steeve pour Steve).

Un problème de plus en plus fréquent souligné par Besnard est le choix de prénoms anglais ou celtiques dotés de multiples variantes orthographiques qui obligeront toute sa vie le porteur à épeler son prénom pour éviter les confusions. Est-ce Elliot, Elliott, Eliott ou Eliot? Mallaury, Malaury, Malaurie, Malorie ou Malory? Killian, Kilian, Kyllian ou Kylian? Autant de variantes d'ores et déjà attestées à l'état civil. Mieux encore, Besnard a relevé en 2003 pas moins de 19 graphies différentes pour Tiphaine, 18 pour Tiffany (variante britannique de Tiphaine, étymologiquement issue de Théophanie), 14 pour Prescillia et presque autant pour sa cousine Priscilla... Dans la même veine, se répandent désormais des prénoms dont la prononciation est telle que le genre, masculin ou féminin, n'apparaît plus à première audition et nécessite une épellation (Morgan/ Morganne, Lilian/Lililiane, etc.), avec cette difficulté supplémentaire que surgissent des formes intermédiaires comme Morgann, attribuées tantôt aux filles tantôt aux garçons. Besnard est convaincu que ces flottements dans la déclinaison de l'identité seront préjudiciables aux intéressés.

\section{ENTRE LA LIBÉRATION DE L'ÉTAT CIVIL ET LA PROTECTION DE LA LANGUE, LES INCOHÉRENCES DU LÉGISLATEUR}

Le sociologue éprouve quelques difficultés à prendre position devant de tels phénomènes. Les uns verront dans cette créativité une preuve de la libre appropriation des biens culturels par les classes populaires. Ils applaudiront à la libération totale du marché du prénom stipulée par la loi du 8 janvier 1993. Chacun est désormais libre de choisir un prénom à sa guise pour ses enfants, sans avoir à s'inquiéter de sa provenance ni de son orthographe. Il n'est plus permis à l'officier d'état civil d'intervenir pour normaliser l'écriture des prénoms étrangers (Besnard donne plusieurs exemples de ces interventions, aujourd'hui interdites par les textes mais peut-être encore présentes par le biais de conseils informels). Livré à lui-même depuis la promulgation de cette nouvelle loi, le peuple retrouverait à sa manière la superbe indifférence qui caractérisait déjà les milieux aristocratiques de la fin du XVIII ${ }^{e}$ siècle face au travail de standardisation de l'orthographe accompli par les imprimeurs: «je plains les gens qui ne savent écrire un mot que d'une seule façon », disait avec dédain le duc de Rohan. Qu'aurait-il dit en découvrant de quelle façon l'école du $\mathrm{XIX}^{\mathrm{e}}$ et du $\mathrm{XX}^{\mathrm{e}}$ siècle allait sacraliser l'orthographe?

Mais un autre point de vue est possible. Il reviendrait à pointer l'incohérence des autorités devant le phénomène de l'américanisation des biens culturels. Les indications livrées par Besnard l'attestent: la libéralisation totale du choix du prénom à l'état civil a relancé le mouvement d'américanisation des prénoms et ouvert la voie au foisonnement des variantes graphiques. Or la même année, étrangement, le ministère de la Culture annonçait la préparation de la loi sur le respect de la langue française dans l'espace public, finalement promulguée le 
4 août 1994. Connu sous le nom de loi Toubon, ce texte fait obligation, sous peine d'amende, d'utiliser le français dans les actes de la vie sociale, commerciale et intellectuelle. Il impose aux personnes morales de droit public et aux personnes privées accomplissant un service public d'utiliser les équivalents français de termes étrangers édictés par les commissions officielles de terminologie. La loi Toubon conforte les dispositions de la loi Bas-Lauriol du 31 décembre 1975, qui imposait déjà de libeller en français la désignation, l'offre, la présentation, la publicité et les modes d'emploi d'un article ou d'un produit. Un arrêté de 1995 a autorisé les associations de défense de la langue française à se porter partie civile contre les contrevenants. Ainsi a-t-on pu voir le législateur, à peu d'intervalle, libérer sans entraves l'américanisation des prénoms tout en interdisant celle des enseignes de magasin ou des papiers à en-tête...

Incohérence? Le législateur n'avait sans doute pas songé que le prénom pouvait être aussi un fait de langue, ni imaginé qu'il se rattachait par bien des traits à l'univers des marques et des enseignes (dont beaucoup prennent d'ailleurs directement la forme de prénoms : Colette, Tiffany's, Jennifer, Agnès B., etc.). Un autre obstacle a contribué à occulter le parallèle entre l'appellation des personnes et l'appellation des biens, à savoir l'intervention du Conseil constitutionnel. Ce dernier a censuré partiellement la loi Toubon le 29 juillet 1994 au nom de la libre communication des pensées et des opinions, proclamée par la Déclaration des droits de l'homme de 1789. L'État, a-t-il expliqué, ne saurait imposer aux personnes privées l'usage d'un français qu'il codifierait lui-même. Il ne peut le faire qu'à l'égard des organismes officiels ou des personnes privées officiant dans l'accomplissement d'un service public. Dans ces conditions, ceux qui voudraient étendre aux prénoms les obligations de la loi Toubon relative à l'emploi du français se heurteraient à une barrière infranchissable: le prénom est un élément personnel de l'identité laissé à la libre appréciation des personnes privées, sans retour possible vers une régulation par l'Etat. Ce double mouvement d'individualisation et d'institutionnalisation est une illustration parmi d'autres d'un paradoxe fondamental souligné en son temps par Durkheim. Je me contente ici de notations rapides. Le sujet mériterait d'être creusé d'un double point de vue juridique et sociologique.

\section{DILEMMES DE PARENTS}

Une grande force du Besnard-Desplanques est de révéler l'existence simultanée des trois modèles de prénomination - diffusion verticale et cyclique, attachement de la bourgeoisie aux prénoms classiques et attrait du peuple pour les prénoms à consonance anglo-saxonne - tout en laissant aux parents leur faculté de choix. Mais il court en filigrane à travers le guide l'idée qu'il est préférable d'éviter les deux polarisations, bourgeoise et populaire. Mieux vaut jouer le jeu de la mode, à condition de le faire en connaissance de cause. Les parents avisés comprennent qu'ils peuvent éviter à leurs enfants les stigmates sociaux qui ne manqueront pas de s'attacher à des prénoms à cycle trop court ou trop long, à des prénoms qui trahissent l'ignorance d'un côté ou l'arrogance de l'autre.

La cote des prénoms pose ainsi à la conscience sociale des parents des dilemmes qui sont exposés de façon amusante dans un roman d'Emmanuel Carrère, Hors d'atteinte? (1988). On y voit un couple d'enseignants divorcés, 
dont le fils se prénomme Quentin, discuter avec des amis qui attendent un enfant. La mère achète un guide sur les prénoms qui n'est autre que le BesnardDesplanques. Elle découvre qu'il est vain d'associer un contenu psychologique ou moral à un prénom. «Forte de ces révélations, elle comptait que le livre l'aiderait à baptiser l'enfant à naître d'un prénom qui, compte tenu des tendances de sa génération, serait original mais pas trop, distingué mais sans prétention». Très vite, elle comprend qu'il faut éviter les «prénoms anglo-saxons récemment importés, choix typiquement prolétarien», mais qu'il ne faut pas «tout de même pas» se tourner vers Arnaud ou Thibaut. «Le plus sûr, trancha Marie-Christine, ce sont des prénoms simples, classiques, sans prétention [...] Pierre ou Paul. Ou Étienne: pas Stéphane, qui pourtant veut dire la même chose, mais Étienne, c'est joli». Sa sœur juge cependant que «c'est un peu facile, ce genre de prénom indémodable», une façon d'éluder le jeu de la mode. Elle pense reprendre à son compte la stratégie qui avait permis de choisir Quentin. Ce qui lui vaut cette réplique: «Tu t'es dit que c'était joli et original, d'accord. N'empêche, j' ai vérifié dans le livre: c'est le type même du prénom donné à un premier enfant par un couple de la région parisienne, aisé, profession libérale, au début des années 80. Comme tous les prénoms qui se finissent en -in, d'ailleurs: Fabien, Damien, Valentin, Adrien... Alors, tu vois.» En définitive, les divers modèles présentés par le guide sont passés en revue et tour à tour écartés, parce qu'il s'avère impossible de faire son choix sans se voir objectivé dans une caractérisation sociale ressentie comme désenchanteresse et cruelle: «Mon pauvre gamin, bêtifia-t-elle en le cajolant. Mon pauvre premier enfant typique de couple-aisé-région-parisienne-débutdes-années-80 - et, tout en serrant Quentin dans ses bras, elle se sentit d'un coup horriblement triste, triste et désarmée [...]».

J'avais souligné cette difficulté dans ma recension de 1987. Chacun entretient une relation enchantée avec son prénom ou le prénom de ses enfants. Comment la préserver si le choix du prénom repose sur les révélations d'une statistique sociale qui risque de rompre l'enchantement?

Je vois une autre difficulté dans l'activité de conseil proposée par l'ouvrage. La volonté de prévenir le stigmate social qui s'attache à certains prénoms risque de poser des problèmes éthiques si elle devient systématique. Quand on recommande à une victime potentielle de prévenir les risques de stigmatisation en adoptant des caractéristiques moins voyantes - par exemple un prénom qui ne trahira ni ses origines sociales ni l'ignorance de ses parents, on accomplit un geste louable de protection des intérêts de l'individu, mais on peut être amené à justifier à l'avance les discriminations sociales sur la base du nom qu'il faudrait plutôt s'employer à combattre. C'est par un raisonnement analogue que les familles juives ont été nombreuses après la Libération à demander la francisation de leur nom de famille et à préférer des variantes françaises de prénoms juifs (comme Mireille pour Myriam), de même que l'on a pu voir des familles harki franciser les prénoms de leurs enfants après 1962 (en substituant, par exemple, Alain à Ali). Or les temps ont changé. Ce n'est plus au stigmatisé que l'on demande de se remettre en cause pour éviter la stigmatisation, mais au stigmatiseur. Il ne suffit plus de vouloir confiner les signes d'appartenance dans la sphère privée pour les rendre tolérables. Tolérer un marqueur d'identité à condition de ne plus le voir, c'est le contraire de la tolérance.

On peut donc s'interroger. Est-il finalement si gênant de voir Chloé converti en Cloé, Timothée en Timothé ou même Théo en Théau? Les aberrations 
graphiques ou linguistiques qui nous choquent tant aujourd'hui ne seront-elles pas tolérées à force de temps avant d'être totalement assimilées? L'histoire nous en montre mille exemples. Il suffit de songer aux avatars qu'ont subis les prénoms les plus classiques en passant les frontières ou en traversant les parlers populaires: troncatures initiales ou finales (aphérèses et apocopes, selon la terminologie des linguistes), amuïssement ou disparition de lettres, changement de voyelles, addition de diminutifs ou d'augmentatifs, etc. L'exemple le plus fameux, bien étudié par les chercheurs allemands, est celui de Nicolas, nom d'un saint byzantin, qui a donné pas moins d'une centaine de variantes répertoriées dans les langues d'Europe, dont les plus connues sont Nicolaus, Nichols, Miklas, Nicolaï, Nicolo, Nicolet, Nick, Nico, Colas, Colin, Collineau, Claus, Klaas, les unes restant des prénoms tandis que les autres subsistent uniquement comme noms de famille. Et ne parlons pas des variantes féminines de Nicolas: Coline, Colette, etc.. Besnard cite lui-même en passant les nombreuses variantes d'Élisabeth (Élisa, Élise, Lise, Lisa, Isabelle, Elsa, Lisbeth, Babette, Betty...), dont la plupart sont érigées aujourd'hui en prénoms autonomes. Que dire enfin du classique François, devenu Francisco en espagnol et, de là, Pancho, Paco, Paquito ou Paquirri, au point de devenir méconnaissable?

Mais ne poussons pas le raisonnement trop loin. À ceux qui ne voient pas l'intérêt de mettre les parents en garde sur les formes de prénom indésirables, Besnard a d'ores et déjà répondu. Il ambitionne seulement de livrer des conseils pratiques à des parents qui demeurent libres de leur choix. Fort de sa colossale banque de données, le sociologue est en mesure de leur livrer des indications sur l'existence des variantes d'un même prénom et sur leur répartition actuelle (pour s'en tenir à cet exemple), toutes informations qui ne sont pas d'emblée disponibles dans le public. Aux parents d'en tirer la conclusion qui s'impose: soit accepter une variante marginale ou extravagante en faisant le pari que leur enfant pourra en assumer les conséquences dans les relations quotidiennes, soit se replier sur la variante centrale, voire sur un prénom plus classique, s'ils préfèrent neutraliser à l'avance les réactions d'autrui. L'apport de La cote des prénoms est d'offrir aux consommateurs une information qui leur permette d'effectuer leurs propres choix en connaissance de cause. La plupart des parents sous-estiment le problème des variantes graphiques et n'anticipent guère les difficultés de présentation de soi que leur enfant risque de rencontrer à l'âge adulte. Les voici désormais prévenus et sensibilisés. À eux de réagir si bon leur semble.

\section{QUAND LA COTATION FAIT LE MARCHÉ}

Il faut toutefois se demander ici si la fraction la plus populaire du public est réellement demandeuse d'une telle information. Après tout, elle fait la loi chez elle à défaut de donner le ton. Pourquoi se soucierait-elle des frilosités du bourgeois? Ne lui suffit-elle pas de recevoir en priorité l'approbation esthétique de l'entourage? Seule une frange désireuse de progresser dans l'échelle sociale et d'anticiper l'ascension de ses enfants par des prénoms bien choisis serait susceptible de s'investir suffisamment dans le jeu onomastique pour acquérir le BesnardDesplanques et en faire son livre de chevet. De fait, une telle minorité n'a rien d'invraisemblable sur le plan sociologique. Elle a son équivalent bien attesté dans 
ces couches sociales qui ont délibérément renoncé à transmettre les langues régionales ou les langues d'immigration à leurs enfants dans l'espoir que, débarrassés de leur accent et de leurs solécismes, ils puissent accroître leurs chances de réussite scolaire et passer plus facilement un jour les concours professionnels. Nul doute qu'un tel calcul de socialisation anticipée puisse également inspirer le choix du prénom.

À ce propos, Besnard fait l'hypothèse raisonnable que la tension entre originalité et conformisme ne couvre pas le même intervalle pour tous les parents: «certains, par leur âge, leur position sociale, leur profession, perçoivent mieux que d'autres l'évolution du goût collectif » (La cote... 2004, p. 12). De façon générale, les parents ont une «conscience confuse» des mécanismes de la ronde des prénoms (p. 309), mais Besnard doute que le flair social des plus avisés éclaire suffisamment leur choix. Il veut croire, non sans optimisme, que son guide rétablira entre tous les publics une égalité d'information (p. 12). Encore faut-il que la probabilité d'acquérir le guide soit également répartie, ce qui suppose au préalable que l'on ait conscience de l'existence d'un problème et qu'on imagine même qu'il puisse trouver sa solution dans un guide. L'exposition du livre dans les librairies d'hypermarchés atténue sans doute cette objection mais ne la lève pas. Soyons réalistes: il est vraisemblable que le guide des prénoms proposé par Besnard et Desplanques aiguise surtout la sensibilité sociale des consommateurs de prénoms déjà sensibilisés au problème. Auquel cas, il contribue davantage à creuser les inégalités d'information qu'à les combler. N'en faisons pas grief aux auteurs: c'est le dilemme insoluble de toute information objective sur le fonctionnement des marchés ou des systèmes. Le ministère de l'Éducation nationale rencontre le même problème quand il tente de diffuser les indicateurs de performance des lycées: l'information distribuée profite d'abord aux parents d'élèves qui cherchent à contourner les obligations de la carte scolaire. Les auteurs de palmarès qui reprennent la même information pour en faire une diffusion plus commerciale ne résolvent pas davantage le problème.

Le travail de Besnard et Desplanques se range, en effet, dans la série grandissante des palmarès qui se font fort de guider le consommateur et l'usager: palmarès des établissements scolaires, palmarès des hôpitaux, palmarès des grandes villes de France, palmarès des régions, etc. On a toujours édité des guides pour éclairer le voyageur amateur de biens culturels (les Grecs et les Romains en étaient déjà friands), les critiques ont dressé de longue date des catalogues ou des argus qui mettent à jour la cotation des valeurs esthétiques, comme le fait la Bourse pour les valeurs industrielles et financières. Le phénomène a pris une ampleur nouvelle avec le développement de l'évaluation, sans doute l'un des phénomènes les plus marquants de la dernière décennie. Nous étendons désormais aux institutions l'exigence d'avoir à fournir des indicateurs objectifs de performance. Mais en l'occurrence, le guide des prénoms présenté par Besnard et Desplanques ne vise aucune cible institutionnelle et n'émet pas de jugement esthétique; il se contente de dresser la «cote» des prénoms telle qu'elle résulte de l'agrégation des choix individuels enregistrés dans l'année. Il ne s'agit pas d'évaluer des performances mais de révéler des préférences.

La meilleure comparaison est encore celle de la cote du marché immobilier. À ce détail essentiel qu'il n'y a pas réellement d'offre et de demande. On ne peut assimiler le calendrier des saints ou le calendrier des Postes à une offre de 
prénoms en bonne et due forme. En fait, ne pourrait-on pas dire que ce sont les guides de prénoms eux-mêmes qui contribuent à créer un début d'offre? Et sur ce point comme sur d'autres, La cote des prénoms est imbattable. Besnard critique à juste titre les guides concurrents qui continuent d'encombrer leurs inventaires de prénoms tirés des calendriers officiels, alors qu'ils ont disparu de la circulation depuis belle lurette ou n'y ont même jamais été présents. En ce sens, il n'est pas exagéré de dire que le marché du prénom commence à exister seulement depuis que La cote des prénoms le fait exister. La formule désormais fameuse de Besnard, selon laquelle «le prénom est un bien gratuit dont la consommation est obligatoire » montre assez qu'il ne s'agit pas initialement d'un marché où se croisent une offre et une demande. Au reste, les prénoms les plus cotés sont justement ceux qui n'ont pas la cote auprès des lecteurs désireux d'éviter les choix conformistes ou trop vulgarisés, lesquels doivent sans doute constituer la majorité des lecteurs.

La formule de Besnard vaut plus généralement pour le choix des mots en général. Il nous faut bien utiliser les mots de la langue si nous voulons communiquer: les mots sont gratuits et l'on ne peut s'en passer. Mais le répertoire des mots est un réservoir sans fond. On y trouve de tout, des tournures les plus usées aux improvisations les plus téméraires, et à tout instant il faut choisir. C'est lorsqu'ils sont «choisis » que les mots prennent de la valeur et, valorisent ou dévalorisent ceux qui les emploient. Le problème spécifiquement traité par Besnard, c'est que votre prénom a été choisi une fois pour toutes et choisi par d'autres que vous, sans possibilité de reprise ou à peine. Pour celui qui le porte, le prénom est toujours en retard d'une génération.

On rejoint ici une des difficultés majeures de la théorie du choix rationnel: dans nombre d'univers sociaux, l'agent rationnel n'est guère autorisé à refaire ses calculs, alors que le contexte pris en compte dans ses calculs a changé. Du coup, un choix initialement rationnel cesse de l'être si l'horizon temporel nécessaire au calcul du rendement de l'investissement est trop lointain. Sur le modèle des artistes qui prennent à l'âge mûr un pseudonyme «jeune» (un exemple souvent évoqué par Besnard pour illustrer la valeur sociale du prénom), on pourrait imaginer un monde où le choix des prénoms ne serait pas seulement libéré au moment de la naissance mais également réactualisable à tout instant au cours de la vie: le prénom attribué par les parents ne serait qu'un prénom d'attente, chacun pourrait ensuite choisir le sien à sa guise et le renouveler librement en cas de besoin. C'est seulement dans ce cas, d'ailleurs, qu'il serait réellement légitime de parler de «consommation» à propos des prénoms. On pourrait même faire sauter un ultime verrou en autorisant chacun à porter simultanément plusieurs prénoms ou à multiplier les pseudonymes selon ses interlocuteurs. Ce genre de pratique a d'ailleurs des précédents dans certaines sociétés traditionnelles d'Amazonie, d'Afrique ou du Grand Nord et même, à un certain degré, dans le système des hypocoristiques et des surnoms pratiqués par certaines communautés rurales d'Europe. Le caractère obligatoire du prénom serait ainsi limité à l'obligation d'avoir au moins un prénom à un moment donné.

Solution irréaliste, bien sûr, car elle conviendrait seulement à des univers de petite taille où l'interconnaissance à vue suffit à garantir la stabilité des identités (ce qui est un peu le cas des milieux artistiques chez nous). Dans les vastes sociétés où nous évoluons, nous avons besoin d'identifiants stables pour décliner 
notre identité devant des inconnus ou voir notre dossier personnel correctement suivi par les institutions. S'il faut conserver le parallèle avec le monde économique, on dira que le prénom ne se situe pas du côté des biens consommables mais du côté des marqueurs d'identité des acteurs (logo, enseigne, nom de marque).

\section{LE SENS ET LE SON}

Le parallèle linguistique incite à revenir sur la question majeure de l'arbitraire linguistique. Les guides que l'on trouve dans le commerce se trompent manifestement quand ils cherchent à remotiver le prénom, au sens saussurien du terme. On veut lui donner un sens, réveiller son étymologie, le rendre signifiant. Mais cet effort contredit les usages les plus ordinaires, y compris lorsque l'étymologie est connue. Les parents qui choisissent d'appeler leur fils Philippe ont beau savoir, pour une fraction d'entre eux, que Philippe signifie «qui aime les chevaux », ce n'est pas là la raison de leur choix. En optant pour Philippe, ils optent pour un prénom classique, indémodable, transnational. Dans leur immense majorité, les prénoms n'ont pas de sens apparent et sont donc parfaitement immotivés, comme l'étaient déjà les prénoms germaniques (Henri, Richard, Bertrand, etc.) quand ils furent adoptés au Moyen Age.

Plus que la sémantique, c'est la phonétique qui compte. Besnard est devenu de plus en plus attentif à ce phénomène. Il décèle des familles sonores de prénoms qui apparaissent et disparaissent en bloc. On connaît la vague des prénoms féminins en -ette dans les années trente: Georgette, Paulette, Yvette, Lucette, Annette, Juliette... D'autres séries marquant plus ou moins fortement le sexe féminin se sont succédé depuis lors: séries en -ie, en -ine, en -iane. La vogue récente des prénoms romains: Fabien, Sébastien, Julien, Damien, Aurélien, Adrien, Cyprien, Victorien, Flavien ou Adrien ne témoigne pas d'un intérêt particulier pour l'Antiquité mais correspond à un engouement pour une famille sonore, que l'accent sur la finale met particulièrement en relief dans notre langue. Des séries d'origine irlandaise ou écossaise (Morgan, Jordan, Yoann, Killian, Dorian...) sont prisées pour leur finale originale. La dernière nouveauté, signalée en 2003, est l'essor des prénoms qui alignent deux voyelles à la suite, dans une série de deux syllabes aussi sommaire qu'un rythme techno: Chloé, Théo, Maë, Léa, Mia, Noah, Tao, Yoan. Ainsi la ronde des prénoms est-elle d'abord une valse des sonorités. Comme nous le disions en 1986, en nous inspirant des théories de Paul Guiraud, un sémantisme diffus peut s'attacher à certaines sonorités (à commencer par les terminaisons féminines, plus ou moins diminutives, ou les terminaisons masculines, plus ou moins énergiques, solennelles, adoucies, voire androgynes, comme on le voit avec la série Morgann, Dorian, Killian, etc.). Cela s'effectue indépendamment du sens intrinsèque ou prétendu tel du prénom. Ces groupes de sonorités reposent davantage sur un pouvoir d'évocation que sur un programme déchiffrable. Elles sont en cela analogues aux impressions musicales en général.

À l'échelle des régions, la dernière édition du Besnard-Desplanques apporte un éclairage inédit sur le destin des sonorités. Elle montre que des mouvements de régionalisation vont de pair avec la propagation d'ondes nationales. Les régions frontalières ou périphériques semblent de plus en plus sensibles aux sonorités des parlers locaux, que l'on a largement cessé de parler et de comprendre et qui s'ap- 
préhendent désormais de plus en plus comme un matériau sonore dont on perçoit les qualités esthétiques. On le voit sur la Côte d'Azur, avec l'apparition de prénoms italiens (Enzo, Lorenzo, Fabio). On l'observe au Pays basque avec la faveur d'un prénom comme Bixente, transcription locale de Vincente. Dans le Roussillon et les Hautes-Pyrénées, avec l'émergence de Pablo. Mais c'est surtout le succès des prénoms bretons qui attire l'attention. On n'a jamais eu autant de prénoms à sonorité celtique (Nowenn, Maïwenn, Gwendal, Gurvan, Titouan...) que depuis la disparition totale de la langue bretonne chez les jeunes générations de parents (les écoles Diwan progressent légèrement d'année en année mais regroupent une infime fraction des enfants). Et ce mouvement va de pair avec le formidable engouement de la région pour la musique celtique. À défaut de transmettre la langue bretonne, les jeunes parents transmettent à leurs enfants un prénom bretonnant et un univers sonore celtisant. Rien de tel en Alsace, où le déclin du dialecte - pourtant attesté - n'en est pas encore arrivé à ce stade. Il faudra voir dans quelques années si la perte annoncée de la langue conduit les Alsaciens à se tourner vers des substituts identitaires analogues à ceux de la Bretagne.

Aux qualités sonores s'ajoutent peut-être, dans certains milieux, des qualités visibles liées à l'apparence graphique du prénom. Non pas l'orthographe étymologisante ou érudite, mais la présence d'un graphème saillant, comme l'est la substitution archaïsante de la lettre y à la lettre i en milieu aristocratique ou bourgeois semi-cultivé et comme peut l'être aujourd'hui la présence d'un x au milieu d'un nom (Besnard signale la série Axel, Maxime, Maxence, Alexandre, Alexis, Alix, Roxane...), qui peut séduire à la fois par sa valeur sonore et sa valeur graphique.

$\mathrm{Au}$ total, le chantier ouvert depuis plus de quinze ans par Besnard et Desplanques multiplie les découvertes en déplaçant les questions. Récapitulons quelques acquis:

- «un prénom n'est ni beau ni laid en soi », ce n'est pas lui qui est en cause mais le moment où on le choisit: avant, avec ou après les autres consommateurs;

- les prénoms sont trop démotivés pour faire sens, ils valent d'abord par leur son; le jugement esthétique de la mode a d'abord pour objet des familles sonores de prénoms;

- le goût des prénoms neufs se diffuse de haut en bas dans le corps social, mais seulement pour une partie d'entre eux, les prénoms populaires et les prénoms bourgeois ayant des origines et des destins de plus en plus séparés, ce qui récuse les explications de la diffusion par de simples effets de domination;

- le cycle complet des prénoms à la mode dure environ 150 ans, soit 40 ans de vie au grand jour et 110 ans de purgatoire, mais ces deux durées restent à portée de la mémoire humaine;

- la stabilité d'un prénom à l'échelle nationale peut couvrir une lente propagation de région en région sur une quarantaine d'années;

- tout en étant prises dans le mouvement cyclique national, certaines régions frontalières ou périphériques, à commencer par la Bretagne, manifestent une grande productivité en matière de pronoms, qui semble compenser le déclin des langues régionales; 
- la législation de 1994 a libéré le choix du prénom dans les milieux populaires, mais au profit d'une hégémonie américaine, tandis que les variantes graphiques d'un même prénom se sont multipliées, comme pour mieux permettre d'individualiser les prénoms partagés avec autrui;

- la mondialisation des prénoms est en marche, mais l'apparition de quelques grands prénoms internationaux venus d'outre-Atlantique n'empêche pas les systèmes nationaux de suivre encore leur propre logique, avec des cycles décalés et inégalement rapides.

On mesure à cette énumération - pourtant incomplète - l'étendue des questions traitées par la petite entreprise Besnard \& Desplanques et son observatoire permanent. Grâce à leurs travaux, nous mesurons mieux combien le choix du prénom est au croisement de tous les axes de l'identité: individu, sexe, génération, groupe social, région, nation, espace européen et transatlantique. De proche en proche, les connaissances produites par Besnard et Desplanques touchent à des pans entiers de la science sociale: sociologie du goût et de la consommation, sociologie de la diffusion et des échanges culturels, sociologie des classifications sociales, histoire de l'immigration, sociolinguistique, sociologie de la famille et de l'identité individuelle. Un sujet de recherche qui pouvait sembler marginal occupe désormais une position centrale dans la discipline. Souhaitons encore longue vie à cette entreprise, qui n’a pas fini de nous livrer ses richesses !

Institut national d'études démographiques (INED), Paris

francois.heran@ined.fr

\section{RÉFÉRENCES BIBLIOGRAPHIQUES}

Besnard Ph., «Pour une étude empirique du phénomène de mode dans la consommation des biens symboliques: le cas des prénoms », Archives européennes de sociologie, 1979, 20: 343-351.

- et G. Desplanques, La cote des prénoms en 2004. Connaître la mode pour bien choisir un prénom, Paris, Balland, septembre 2003, 479 p. [1 $1^{\text {re }}$ éd., Un prénom pour toujours. La cote des prénoms hier, aujourd' hui et demain, Balland, 1986; mis à jour chaque année avec les données de 1'Insee].

- et G. Desplanques, «Les catégories socioprofessionnelles à l'épreuve de la stratification temporelle des goûts », Revue française de sociologie, 1999, 40 (1): 97-109.

- et Cyril Grange, «La fin de la diffusion verticale des goûts? Prénoms de l'élite et du vulgum», L'Année sociologique, 1993, 43: 269-294.

- «The study of social taste through first names: comment on Lieberson and Bell», American Journal of Sociology, mars 1995, 100 (5): 1313-1316.

Carrère E., Hors d'atteinte?, Paris, éd. P. O. L., 1988, pp. 53-58 [rééd. «Folio », Gallimard, 1989. - Où l'on voit les deux protagonistes, une professeur de collège et son ex-mari, commenter longuement le guide de Besnard et Desplanques].

Desplanques G., «Les enfants de Michel et Martine Dupont s'appellent Nicolas et Céline», Économie et statistique, $\mathrm{n}^{\circ} 184$, janvier 1986, pp. 63-83.

Héran F., Compte rendu de: Besnard Ph., Desplanques G., Un prénom pour toujours, Paris, Balland, 1986, in: Revue française de sociologie, 1987, 28 (4): 708-711.

Moreau M., J. Postic et S. Rivière, «Julien Meyer, fils de Jean Meyer », Chiffres pour l’ Alsace, $\mathrm{n}^{\circ} 34$, Strasbourg, Insee, novembre 1996. 